\title{
Capsule Commentary on Goto et al.'s Incidence of Acute Cardiovascular Event After Acute Exacerbation of COPD
}

\author{
Mhd Wasem Alsabbagh, BSc Pharm, PhD \\ School of Pharmacy, University of Waterloo, Waterloo, Canada.
}

J Gen Intern Med 33(9): 1552

DOI: $10.1007 / \mathrm{s} 11606-018-4561-0$

(c) Society of General Internal Medicine 2018

$\mathrm{S}$ ome clinical questions cannot be answered by randomized clinical trials. ${ }^{1}$ As such, harnessing vigorous observational evidence generated from big data to improve clinical care is a major undertaking by researchers. ${ }^{2}$ The article by Goto et al. in this issue represents a significant example of such an endeavor. ${ }^{3}$ It uses population-based data with strong methods to answer a highly relevant clinical question. Although previous studies established a significant association between COPD and cardiovascular diseases (CVD) adverse effects, limited research investigated the association between acute exacerbation of COPD (AECOPD) and CVD outcomes.

Above and beyond the appropriate choice of the cohort of patients who experienced AECOPD to unveil the association with CVD adverse effects, the statistical method of selfcontrolled case series analysis was advantageous in ensuring the robustness of the findings. This method takes into account all individual characteristics that may confound the relationship between exposure and outcome. ${ }^{4}$ Furthermore, the multiple sensitivity analysis enabled the researchers to affirm that the findings are valid. For example, comparing the incidence rate ratios (IRRs) of CVD outcomes (the signal) with the rate of all-cause hospitalizations (the noise) increases the confidence that the findings are substantial and not a result of natural progression of COPD.

The authors speculated several explanations of the findings - including the role of activation of inflammatory cascade in the short-term increase in CVD outcome and the role of interruption of CVD protective modalities in the long-term

Published online July 10, 2018 effects. Regardless of the exact mechanism of this association, the important message to clinicians caring for elderly patients with COPD should be loud and clear: (1) apply evidence-based therapies to avoid AECOPD as much as possible and (2) if patients experience AECOPD, pay special attention to the continuation of cardio-protective therapies to avoid the unintended increase in CVD risk. Withdrawal of cardioprotective medications - such as beta blockers - is still prevalent in contemporary practice, in spite of vast data that support its safety and effectiveness. ${ }^{5}$ Hence, the results of this article would be essential to remind clinicians about the importance of optimizing CVD treatments among patients who present with AECOPD.

Corresponding Author: Mhd Wasem Alsabbagh, BSc Pharm, PhD; School of Pharmacy University of Waterloo, Waterloo, Canada (e-mail: Wasem.alsabbagh@uwaterloo.ca).

\section{Compliance with Ethical Standards:}

Conflict of Interest: The authors declare that they do not have a conflict of interest.

\section{REFERENCES}

1. Barton S. Which clinical studies provide the best evidence? BMJ. 2000; 321(7256): 255-256.

2. Nash D. Harnessing the Power of Big Data in Healthcare. Am Health Drug Benefits. 2014; 7(2): 69-70.

3. Goto T, Shimada YJ, Faridi MK, Camargo CA, Hasegawa K. Incidence of acute cardiovascular event after acute exacerbation of COPD. J Gen Intern Med. https://doi.org/10.1007/s11606-018-4518-3

4. Petersen I, Douglas I, Whitaker $\mathbf{H}$. Self controlled case series methods: an alternative to standard epidemiological study designs. BMJ. 2016;354:i4515.

5. Albouaini K, Andron M, Alahmar A, Egred M. Beta-blockers use in patients with chronic obstructive pulmonary disease and concomitant cardiovascular conditions. Int J Chron Obstruct Pulmon Dis. 2007; 2(4): 535-540. 